\title{
Recherches sur la toxicité et la valeur trypanopréventive du moranylate d'éthidium
}

\author{
II. Solubilité. Toxicité. Propriétés préventives \\ en conditions d'infestation naturelle en République du Cameroun
}

par J. MAGIMEL $†$

Les trypanosomiases ont toujours présenté un grave obstacle à l'élevage des zébus arabes dans la région du Nord-Cameroun correspondant à la préfecture du Logone-Chari.

C'est une région de type sahélien caractérisé par une steppe arbustive théoriquement favorable à l'élevage. Malheureusement de nombreux affluents et défluents du Chari créent, par leur présence et leurs débordements, des conditions favorables à la vie des glossines et à la multiplication de très nombreux arthropodes piqueurs, ce qui rend l'élevage du bétail précaire pendant une certaine partie de l'année. Nous insistons bien sur le fait que dans cette zone l'infestation trypanosomienne est moyenne et $n$ 'interdit pas l'élevage mais provoque chaque année une mortalité importante et surtout une morbidité rendant le bétail très sensible aux autres affections.

C'est pour ces raisons qu'au début de 1956 le Service de l'élevage du Cameroun demanda au Laboratoire de Farcha d'entreprendre une étude approfondie des moyens de lutte contre ces affections. Entre autres expérimentations fut envisagée l'étude de divers trypanocides ou trypanopréventifs connus afin de déterminer celui ou ceux qui se montreraient les plus efficaces pour conserver et développer le troupeau existant.

Ce n'est qu'à la fin de 1956, après l'ouverture de sa section d'entomo-protozoologie, que le Laboratoire de Farcha a pu se charger de ce travail. Le gouvernement du Cameroun participa à son financement en mettant à notre dispo-

Reçu pour publication : février 1961.

Rev. Elev. Méd. vét. Pays trop., 1961, 14, no 2. sition des crédits permettant l'achat des animaux et leur gardiennage.

Le programme de travail suivant fut alors établi :

1) Etude préliminaire sur la solubilité et la toxicité des nouveaux produits utilisés.

2) Ełude des propriétés préventives sur un petit nombre d'animaux.

3) Selon les résultats obtenus, extension de l'expérimentation sur un plus grand nombre d'animaux,

\section{I. - ÉTUDE PRÉLIMINAIRE}

\section{Solubilité}

Lo solubilité du moranylate d'éthidium est à peu près nulle dans l'eau quelle que soit la température. Le titre est limité par le volume pratique à injecter et même au 1/50 en equ distillée, la solubilité est pratiquement nulle. Les quelques solvants organiques essayés n'ont pas donné de résultat. L'alcool éthylique pur ou dilué n'améliore pratiquement pas la solubilisation; les acides et bases dilués sont également sans effet. Le seul mode de préparation en vue de l'injection est la suspension. Au début a été utilisée l'eau distillée seule, mais par la suite, l'emploi de l'eau de robinet non filtrée n'a jamais entraîné de complications septiques. La conservation du mélange eau-moranylate d'éthidium semble aisée, bien que la décantation soit rapide. Si on a soin d'homogénéiser la suspension avant l'emploi, il semble que l'on puisse différer l'injection de plusieurs jours. A la température du laboratoire ou en chambre froide $\left(+4^{\circ} \mathrm{C}\right)$, en pleine lumière ou dans l'obscurité. l'aspect des échan- 
tillons de la suspension n'a pas changé en six mois: 2 phases, une liquide, claire, pratiquement incolore, une pulvérulente, opaque, vineuse. II n'y a ni concrétion, ni floculation visible, ni solubilisation appréciable. La tenue de la suspension avec le temps a pu être évaluée upproximativement, à la température ambiante, par mesure de la hauteur des deux phases dans le flacon d'origine auquel on avait ajouté $10 \mathrm{ml}$ d'eau. L'intérêt pratique d'une telle mesure est limité au fait qu'il s'avère pratiquement impossible d'utiliser un pistolet injecteur à réserve de liquide, la décantation étant trop rapide.

\section{Toxicité}

L'expérimentation a porté sur la toxicité générale et locale en fonction des doses et des voies d'injection, le moranylate d'éthidium étant utilisé en suspension au 1/10 dans l'eau distillée. Nous avons tout d'abord essayé des doses de 5 ef $10 \mathrm{mg} / \mathrm{kg}$ du produit soit, pour des bouvillons de $100 \mathrm{~kg}$, des doses de 0,5 et $1 \mathrm{~g}$ de la suspension injectées à l'encolure, sous la peau ou dans les muscles. Dans ces conditions la toxicité générale est négligeable ou nulle, il n'y a pas d'atteinte de l'appétit, pas d'hyperthermie. En revanche, les réactions locales sont importantes et d'intensité croissante avec la dose : minimes après l'injection intramusculaire, plus fortes après l'injection sous-cutanée. Du type inflammatoire et nécrotique, aux doses de 5 et $10 \mathrm{mg} / \mathrm{kg}$, les réactions n'ont jamais donnć d'ulcération, ni d'abcédation.

D'abord chaud ef odématié, le point d'injection est le siège d'une réaction d'enkystement fibreuse à logettes liquidiennes. L'autopsie révèle qu'à l'intérieur des logettes le liquide est sous tension, clair, rose vineux et chargé de particules rouge foncé plus ou moins amalgamées par de la fibrine. Le volume du dépôt rouge sombre laisse penser a priori que le moranylate d'élhidium n'a pas été résorbé. Ceci fut d'ailleurs confirmé par l'étude histo-pathologique conflée au laboratoire d'anatomie pathologique de l'Ecole vélérinaire d'Alfort (nécrose du tissu musculaire ; réaction inflammatoire aiguë autour du foyer de nécrose, rétention du médicament dans le tissu conjectif intra-musculaire).

Dans une série d'expériences complémentaires, pour tenter d'expliquer les réactions très intenses avec gros délabrement qui avaient été observées à Bouar avec une dose de $15 \mathrm{mg} / \mathrm{kg}$, nous avons utilisé des bouvillons de plus grand format et nous avons forcé les doses initiales jusqu'à $15 \mathrm{mg} / \mathrm{kg}$ (3 g au total de moranylate d'éthidium). Comme à Bouar, nous avons obtenu de grosses réactions non abcédées dans le cas d'injection intramusculaire et des ulcérations sèches suivies de complications septiques secondaires dans le cas d'injection sous-cutanée.

Par ailleurs, des doses de 20 à $50 \mathrm{mg} / \mathrm{kg}$ de moranylate d'éthidium ont été injectées à des bouvillons de petit format de façon à atteindre une dose totale de $3 \mathrm{~g}$ par animal. Les réactions n'ont pas été plus intenses que celles observées précédemment avec une dose de $15 \mathrm{mg} / \mathrm{kg}$ sur des bœufs de $200 \mathrm{~kg}$ (soit une même dose de $3 \mathrm{~g}$ par animal).

Il apparait ainsi que le moranylate d'éthidium, étant très peu soluble, agit comme un corps étranger (et d'ailleurs la réaction nécrotique avec ulcération est bien une réaction d'expulsion). La cicatrisation suit toujours I'ulcération, elle est du type granuleux.

En conclusion, le moranylate d'éthidium est peu toxique, mais provoque des réactions locales qui condamnent l'utilisation par voie souscutanée, surtout dans le cas de boufs de grand format donc de doses absolues élevées $(2 \mathrm{~g})$.

Même par voie intramusculaire ce trypanocide devra être injecté dans une région de valeur secondaire pour la boucherie, c'est-à-dire qu'il faudra proscrire le globe ou les lombes, ef se limiter à l'encolure.

\section{II. - EXPÉRIMENTATION DE RIGGIL}

Dans un premier temps nous avons décidé de conduire notre expérimentation sur un petit nombre d'animaux qui seraient placés à Riggil. Riggil est un Centre vétérinaire situé sur la rive camerounaise du Chari à peu de distance du Laboratoire de Farcha. II a l'avantage non seulement d'être à proximité du Laboratoire de Farcha, mais également de présenter des conditions d'élevage identiques à celles que l'on peut trouver dans toute la région Nord-Cameroun, avec, semble-t-il, une infestation trypanosomienne plus sévère, ce qui ne peut être qu'un avantage pour les expériences. 
TABLEAIJ I. - Efficacité trypanopréventive ef toxicité de différen's trypanocides dans la zone infectée de Riggil

\begin{tabular}{|c|c|c|c|c|c|c|c|}
\hline \multirow[b]{2}{*}{ Produits utilisés } & \multirow[b]{2}{*}{ Dose } & \multirow{2}{*}{$\begin{array}{l}\text { Nbre } \\
\text { d'ani- } \\
\text { maux }\end{array}$} & \multirow{2}{*}{$\begin{array}{l}\text { Dépara- } \\
\text { sitage }\end{array}$} & \multicolumn{3}{|c|}{ Résultats } & \multirow[b]{2}{*}{ Réactions } \\
\hline & & & & Infectés & Morts & $\begin{array}{c}\text { In- } \\
\text { demnes }\end{array}$ & \\
\hline $\begin{array}{l}\text { Moranylate } \\
\text { d'Ethidium }\end{array}$ & $\begin{array}{c}5 \mathrm{mg} / \mathrm{kg} \\
» \\
10 \mathrm{mg} / \mathrm{kg} \\
» \\
15 \mathrm{mg} / \mathrm{kg} \\
»\end{array}$ & $\begin{array}{l}1 \\
1 \\
1 \\
1 \\
1 \\
1 \\
1\end{array}$ & $\begin{array}{l}\text { ovi } \\
\text { non } \\
\text { oui } \\
\text { non } \\
\text { oui } \\
\text { non }\end{array}$ & & $\begin{array}{l}10 \mathrm{e} \text { mois accident } \\
12 \text { mois } 4 \mathrm{j} \text {. acci- } \\
\text { dent } \\
11 \text { mois } 22 \mathrm{j} \text {. acci- } \\
\text { dent } \\
145 \mathrm{e} \text { j. mort ca- } \\
\text { chexie }\end{array}$ & $\begin{array}{l}16 \text { mois } \\
14 \text { mois }\end{array}$ & $\begin{array}{l}\text { Réactions locales } \\
\text { faibles pour doses } \\
\text { inférieures à } \\
10 \mathrm{mg} / \mathrm{kg} \text {. Très im- } \\
\text { portantes à dosage } \\
\text { supérieur lors de } \\
\text { réactions générales }\end{array}$ \\
\hline Prothidium & $\begin{array}{c}2 \mathrm{mg} / \mathrm{kg} \\
》 \\
3 \mathrm{mg} / \mathrm{kg} \\
» \\
4 \mathrm{mg} / \mathrm{kg} \\
\# \\
5 \mathrm{mg} / \mathrm{kg} \\
\#\end{array}$ & $\begin{array}{l}1 \\
1 \\
1 \\
1 \\
1 \\
1 \\
1 \\
1 \\
1\end{array}$ & $\begin{array}{l}\text { oui } \\
\text { non } \\
\text { oui } \\
\text { non } \\
\text { ovi } \\
\text { non } \\
\text { ovi } \\
\text { non }\end{array}$ & $\begin{array}{l}\text { le } 133 \mathrm{ej} . \\
\text { le } 120 \mathrm{e} j . \\
\text { le } 150 \mathrm{e} j . \\
\text { le } 150 \mathrm{e} j \\
7 \text { mois } 5 \mathrm{j} . \\
11 \text { mois } 6 \mathrm{j} \text {. }\end{array}$ & $23 \mathrm{e} \mathrm{j}$. peste & 15 mois & $\begin{array}{l}\text { Réactions locales fai- } \\
\text { bles ou passagères } \\
\text { Pas de réaction géné- } \\
\text { rale }\end{array}$ \\
\hline $\begin{array}{l}\text { Antrycide } \\
\text { Pro Salt }\end{array}$ & $\begin{array}{l}3 \mathrm{~g} \\
3 \mathrm{~g}\end{array}$ & $\begin{array}{l}2 \\
2\end{array}$ & $\begin{array}{l}\text { oui } \\
\text { oui } \\
\text { non } \\
\text { non }\end{array}$ & 11 mois $20 \mathrm{j}$. & $30^{\mathrm{e}} \mathrm{j}$. peste & $\begin{array}{l}15 \text { mois } \\
16 \text { mois }\end{array}$ & $\begin{array}{l}\text { Réactions locales fai- } \\
\text { bles }\end{array}$ \\
\hline Trypadine & $\begin{array}{c}1 \mathrm{mg} / \mathrm{kg} \\
" \\
1 \mathrm{mg} / \mathrm{kg} \\
》\end{array}$ & $\begin{array}{l}1 \\
1 \\
1 \\
1\end{array}$ & $\begin{array}{l}\text { oui } \\
\text { oui } \\
\text { non } \\
\text { non }\end{array}$ & $\begin{array}{l}\text { le } 40 \mathrm{e} \mathrm{j.} \\
\text { ile } 90^{\mathrm{e}} \mathrm{j} . \\
\text { le } 86^{\mathrm{e}} \mathrm{j} . \\
\text { le } 86 \mathrm{e} \mathrm{j} .\end{array}$ & . & & Pas de réaction \\
\hline Naganol & $\begin{array}{c}10 \mathrm{mg} / \mathrm{kg} \\
》\end{array}$ & $\begin{array}{l}1 \\
1\end{array}$ & $\begin{array}{l}\text { oui } \\
\text { non }\end{array}$ & $\begin{array}{l}\text { le } 26^{\mathrm{e}} \mathrm{j} \\
\text { le } 25^{\mathrm{e}} \mathrm{j}\end{array}$ & ; & & Pas de réaction \\
\hline $\begin{array}{l}\text { Moranylate } \\
\text { de lomidine }\end{array}$ & $\begin{array}{c}5 \mathrm{mg} / \mathrm{kg} \\
》 \\
10 \mathrm{mg}^{\prime} \mathrm{kg} \\
》 \\
15 \mathrm{mg} / \mathrm{kg} \\
》\end{array}$ & $\begin{array}{l}1 \\
1 \\
1 \\
1 \\
1 \\
1\end{array}$ & $\begin{array}{l}\text { oui } \\
\text { non } \\
\text { oui } \\
\text { non } \\
\text { oui } \\
\text { non }\end{array}$ & 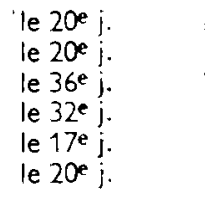 & 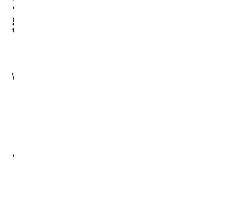 & & Pas de réaction \\
\hline Ethidium & $\underset{\nu}{1 \mathrm{mg} / \mathrm{kg}}$ & $\begin{array}{l}1 \\
1\end{array}$ & $\begin{array}{l}\text { ovi } \\
\text { non }\end{array}$ & $\begin{array}{l}\text { le } 90^{\circ} \mathrm{j} \\
\text { le } 40^{\circ} \mathrm{j} .\end{array}$ & & & Pas de réaction \\
\hline Témoins & & 14 & non & $\begin{array}{l}\text { entre le } 11 \text { e ef } \\
\text { le } 35 \text { e jour }\end{array}$ & & & \\
\hline
\end{tabular}

Dans toute la zone de pâturages entourant Riggil ainsi que dans tout le Nord-Cameroun les principaux insectes piqueurs rencontrés sont: Glossina tachinoides-Ancala fasciata-Tabanus taeniola et Atylotus agrestis. Quant aux trypanosomes en cause nous avons trouvé : Trypanosoma vivax, Trypanosoma congolense et Trypanosoma bruce!.

\section{Conduite de l'expérimentation}

Animaux: Quaranie-six bouvillons zébu arabe de 1 à 2 ans ont servi à l'expérimentation après que nous soyons assurés qu'ils étaient indemnes de trypanosomes. Ils ont été répartis en 8 lots d'importance variable selon le produit et les. 
doses utilisés. Dans chaque lot, la moitié des animaux a été déparasitée.

Produits utilisés: En dehors du moranylate d'éthidium et du Prothidium nous avons utilisé comme produits de référence ::

Antrycide (Prosalt); Trypadine; Naganol ; Ethidium ; Moranylate de Lomidine.

Mode d'emploi : la Trypadine, le Naganol et l'Éthidium ont été utilisés aux doses et par les voies classiques. Le Prothidium a été utilisé par voie intramusculaire aux doses de 2,3,4 et $5 \mathrm{mg} / \mathrm{kg}$. Quant au moranylate d'éthidium il a été injecté également par voie intramusculaire aux doses de 5,10 et $15 \mathrm{mg} / \mathrm{kg}$ en suspension aqueuse à 10 p. 100.

Durée de l'expérience : Commencée le 3 juillet 1957, elle a été poursuivie jusqu'en septembre 1958.

Témoins: Renouvelés dès qu'ils étaient trouvés infestés : I'infestation se fait entre le $11^{\mathbf{e}}$ et le 35 e jour.

Contrôle : Deux fois par semaine les deux premiers mois, toutes les semaines les mois suivants.

Résultats : Résumés dans le tableau 1.

Commentaires: Trois produits se sont montrés trypanopréventifs.

- L'Antrycide (Prosalt) qui a protégé les animaux au moins 11 mois et 20 jours (à signaler qu'à la suite d'unc erreur, les bouvillons ont reçu une dose triple de ce qui est habituellement utilisé).

- Le Prothidium qui, à la dose de $5 \mathrm{mg} / \mathrm{kg}$, a protégé les animaux au moins 11 mois 6 jours.

- le Moranylate d'Ĺthidium qui a protégé les animaux jusqu'à 16 mois à une dose de $5 \mathrm{mg} / \mathrm{kg}$.

La Trypadine ef l'Éthidium ont assuré une protection d'une durée prévue; le Naganol et le Moranylate de Lomidine n'ont pratiquement eu aucune action.

Le fait de déparasiter les animaux n'a modifié en rien le temps de protection.

\section{III. - EXPÉRIMENTATION DE SULFA}

Début 1958, c'est-à-dire six mois après le début de l'expérimentation de Riggil, nous avons pensé que les résultats obtenus avec le moranylate d'éthidium étaient suffisamment intéressants pour que l'expérimentation soit reprise sur un plus grand nombre d'animaux et dans les vraies conditions de brousse.

Afin de réduire les frais de l'expérimentation, nous avons procédé de la façon suivante : nous avons d'abord recherché un village où les pertes par trypanosomiase étaient connues et très importantes chaque année; puis nous nous

TABLEAU 11. - Activité curative du Prothidium et trypanopréventive du Moranylate d'Ethidium Expérimentation dans la zone infestée de Sulfa

\begin{tabular}{|c|c|c|c|c|c|c|}
\hline Produits utilisés & Dose & $\begin{array}{l}\text { Nbre } \\
\text { d'ani- } \\
\text { maux }\end{array}$ & Infectés & Morts & Indemnes: & Réactions \\
\hline $\begin{array}{l}\text { Prothidium } \\
\text { (curatif) }\end{array}$ & $2 \underset{I M}{\mathrm{mg}} / \mathrm{kg}$ & 40 & $\begin{array}{l}\text { Infectés au départ } \\
\text { T. congolense } \\
\text { T. vivax. } \\
\text { T. brucei. }\end{array}$ & $\begin{array}{l}9 \text { morts-choc try- } \\
\text { panolytique }\end{array}$ & $\begin{array}{l}\text { tous les autres ani- } \\
\text { maux non infec- } \\
\text { tés } 23 \text { mois après }\end{array}$ & $\begin{array}{l}\text { Locales faibles et } \\
\text { passagères } \\
\text { Générale nulle }\end{array}$ \\
\hline $\begin{array}{l}\text { Moranylate } \\
\text { d'Ethidium } \\
\text { (préventif) }\end{array}$ & $10 \mathrm{mg} / \mathrm{kg}$ & 166 & & 30 morts-cachexie & $\begin{array}{l}\text { tous les autres ani- } \\
\text { maux non infec- } \\
\text { tés } 23 \text { mois après }\end{array}$ & $\begin{array}{l}\text { Très fortes dans } \\
90 \% \text { des cas. }\end{array}$ \\
\hline Témoins & & 66 & $\begin{array}{r}6 \text { en } 1 \text { mois } \\
+4 \text { en } 2 \text { mois } \\
+3 \text { en } 3 \text { mois } \\
+1 \text { en } 4 \text { mois } \\
+1 \text { en } 5 \text { mois } \\
+1 \text { en } 6 \text { mois } \\
+2 \text { en } 9 \text { mois }\end{array}$ & $\begin{array}{l}\text { Traités à l'Ant } \\
\text { de } 20 \mathrm{mg} / \mathrm{kg}\end{array}$ & $\begin{array}{l}\text { rycide à raison } \\
g\end{array}$ & \\
\hline
\end{tabular}


sommes entendus avec les éleveurs qui ont accepté que nous expérimentions nos produits à condition que nous remboursions la valeur de tous les animaux qui mourraient des suites de l'expérience.

\section{Conduite de l'expérimentation}

Animaux utilisés : Zébus arabes de tous âges constituant le troupeau du village de Sulfa, soit : 272 bêtes.

Contrôle préliminaire : les animaux ont été soumis à un examen détaillé (examens directs renouvelés et gouttes épaisses) ce qui a permis de diviser le troupeau en deux lots; 40 trypanosomés (par T. congolense, $T$. vivax, $T$. brucei et association congolense-vivax, brucei-congolense et vivax-brucei) et 232 animaux indemnes. Ce contrôle préliminaire confirma donc l'importance de l'infestation trypanosomienne.

Produits utilisés : Ne possédant pas une quantité suffisante de moranylate d'éthidium nous avons décidé de réserver ce produit à une partie des animaux indemnes en l'utilisant à la dose de $10 \mathrm{mg} / \mathrm{kg}$ en intramusculaire dans l'encolure et de traiter les trypanosomés au Prothidium (à la dose de $2,5 \mathrm{mg} / \mathrm{kg}$ en intramusculaire dans l'encolure).

Témoins : Parmi les animaux indemnes, seuls 166 ont été traités au moranylate d'éthidium ; les 66 autres ont servi de témoins.

Durée de l'expérimentation : Elle a commencé le 22 mai 1958 et est toujours en cours.

Contrôles : Tous les mois les 5 premiers mois pour les témoins. Ensuite tous les trois mois pour tous les animaux.

Résultats : Ils sont résumés dans le tableau II.

\section{Remarques}

Les réactions dues au Prothidium sont faibles (ne pas tenir compte des neuf morts dus au choc trypanolytique). Avec le moranylate d'éthidium elles ont été très fortes chez 90 p. 100 des animaux. Trente de ceux-ci ont présenté une réac- tion locale si volumineuse que, ne pouvant plus fléchir l'encolure, ils ne paissaient plus. Devenus cachectiques, ils ont dû être abattus.

II est intéressant de constater, une fois de plus, que les résultats obtenus sur un très petit nombre d'animaux ne peuvent pas être transposés lorsque l'on travaille sur un grand nombre d'animaux car les conditions de traitement, de surveillance et d'entretien ne sont plus les mêmes. Ainsi dans l'expérience de Riggil, dans les délais normaux, nous n'avions eu qu'un animal mort cachectique après traitement à la dose de $15 \mathrm{mg} / \mathrm{kg}$, et les réactions locales n'avaient été considérées comme vraiment trop fortes qu'à cette dose.

Les témoins, qui se sont infectés pendant les neuf premiers mois de l'expérience et qui ont été traités à l'Antrycide, sont restés ensuite indemnes ainsi que tous les autres animaux d'expérience jusqu'au dernier examen effectué le 19 avril 1960 soit 23 mois après.

Vingt-trois mois après l'injection, les animaux traités au moranylate d'éthidium présentaient toujours une réaction locale importante mais qui ne les gênait pas et ne les empêchait pas de se maintenir en excellent état d'embonpoint.

Conclusions : En zone sahélienne le moranylate d'éthidium a montré des qualités trypanopréventives indiscutables. Malheureusement ce produit doit être utilisé avec précaution car dans les conditions de la brousse, il provoque des réactions locales trop violentes pour que son emploi soit généralisé.

11 mériterait cependant d'être expérimenté à nouveau dans les mêmes conditions que celles de Sulfa, mais à une dose plus faible.

Nous remercions ici les Docteurs DUCROZ et RENARD, successivement chefs du Secteur vétérinaire du Logone-Chari, qui nous ont aidé à mettre sur pied cette expérience ainsi que celle de Sulfa et à assurer la surveillance des animaux d'expérience.

\footnotetext{
Institut d'élevage

et de médecine vétérinaire des poys tropicaux: Laboratoire de recherches vétérinaires de Farcha, Fort-Lamy (Tchod)
} 


\section{RÉSUMÉ}

Le moranylate d'éthidium, utilisé en suspension dans l'eau à 10 p. 100 à des doses inférieures ou égales à $10 \mathrm{mg} / \mathrm{kg}$, est un excellent trypanopréventif qui, utilisé avec précaution dans des conditions de laboratoire, provoque des réactions locales fortes mais acceptables. Par contre, dans les conditions de la brousse, les réactions sont trop violentes pour permettre la généralisation de son emploi.

\section{SUMMARY}

Studies on Ethidium moranylate. II. Solubility. Toxicity. Preventive properties under the natural conditions of infestation in the Cameroon Republic

Ethidium moranylate in a $10 \%$ aqueous suspension at a dose equal to slightly, less than a rate of $10 \mathrm{mg} / \mathrm{kg}$ is an excellent «trypanopreventive » which under laboratory conditions produces wellmarked, though acceptable local reactions. Under field conditions however, on the contrary, the reactions are too heavy to allow of general use.

\section{RESUMEN \\ Investigaciones sobre el moranilato de etidion \\ II. Solubilidad. Toxicidad. Propiedades preventivas en condiciones de infestación natural en la República de Camerun}

El moranilato de etidion utilizado en suspensión en el agua al $10 \%$ a dosis inferiores o iguales a $10 \mathrm{mg} / \mathrm{kg}$ es un excelente preventivo de la tripanosomiasis que, utilizado con precaución en las condiciones de laboratorio, provoca reacciones locales fuertes pero aceptables. En las condiciones del campo las reacciones son demasiado vialentas para permitir la generalización de su empleo. 